\title{
Online Password Guessing Attacks by Using Persuasive Cued Click Points
}

\author{
Priyanka A. Dadmode1, Kiran I. Devadkar² \\ ${ }^{1}$ Department of Computer Engineering, Sharad Institute of Technology Polytechnic, Yadrav, India
}

\begin{abstract}
Graphical Password essentially uses images or representation of images as password. Human brain is good in remembering picture than textual character. This paper work merges pass points, cued click points and persuasive cued click points. The major goal of this work is to reduce the guessing attacks as well as encouraging users to select more random, and difficult passwords to guess. In this paper, the selection of click points depends upon the method selected by user, selection of sound. Sound gives the security for right click points.
\end{abstract}

Keywords: Authentication, graphical passwords, guessing attacks

\section{Problem Description}

The problem associated to vulnerability to shoulder surfing is a major flow that needs to be addressed for current authentication system including graphical password authentication system and traditional alphanumeric password authentication system. According to Webopedia Computer Dictionary, ,Shoulder surfing refers to a direct observation, such as looking over a person's shoulder, to obtain information." As define, shoulder surfing usually happen in busy and crowed place, where the person standing behind would try to peek over your back in order to obtain private Motivation of Research Weakness of Textual Password.

So we implement graphical password method. In this paper, the selection of click points depends upon the method selected by user, selection of sound. Sound gives the security for right click points.

\section{Introduction}

An important usability goal for authentication systems is to support users in selecting better passwords. Users often create memorable password that are easy for attackers to guess, but strong system-assigned password are difficult for users to remember. Graphical passwords essentially use images or representation of images as password. Human brain is good in remembering picture than textual character. The major goal of this work to reduce the guessing attacks as well as encouraging users to select more random, and difficult password guess. Well known security threats like brute force attacks and dictionary attacks can be successfully abolished using this method. The knowledge based techniques are the most wanted techniques to improve real high security i.e. Recognition based and recalls based.

\section{Recall Based Techniques}

There are types of click based graphical password techniques:
1) Pass Points (PP)
2) Cued Click Points(CPP)
3) Persuasive Cued Click Points(PCCP)

\subsection{Pass Point (PP)}

In Pass Points [1], [3], [5] is method where password consists of an ordered sequence of five click-points. User must click within some system-defined region on image.

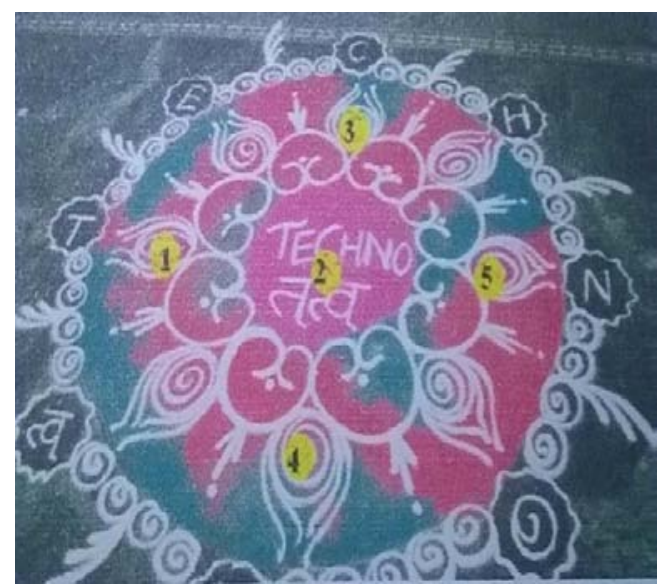

Figure 3.1: Pass Point

\subsection{Cued Click Points(CCP)}

In Cued Click Points [1], [4], [5] method, user selects one point per image for minimum five images. At a time only one image is displayed. When user select click point it goes to next image.

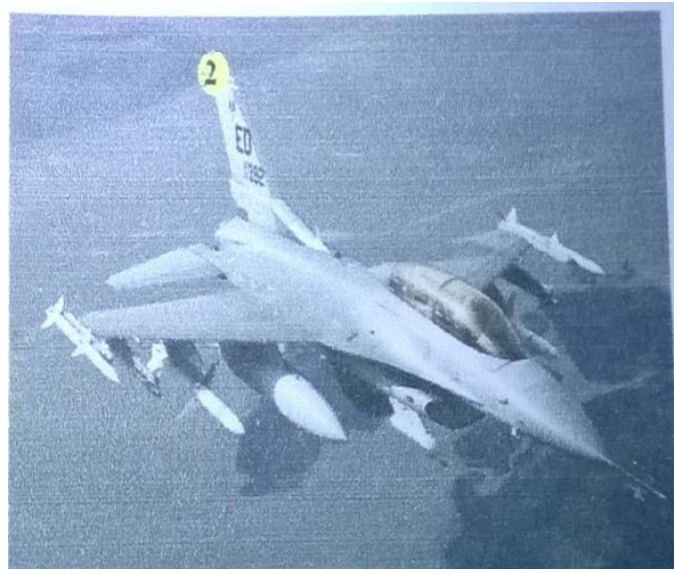

Figure 3.2.1: Cued Click Point 


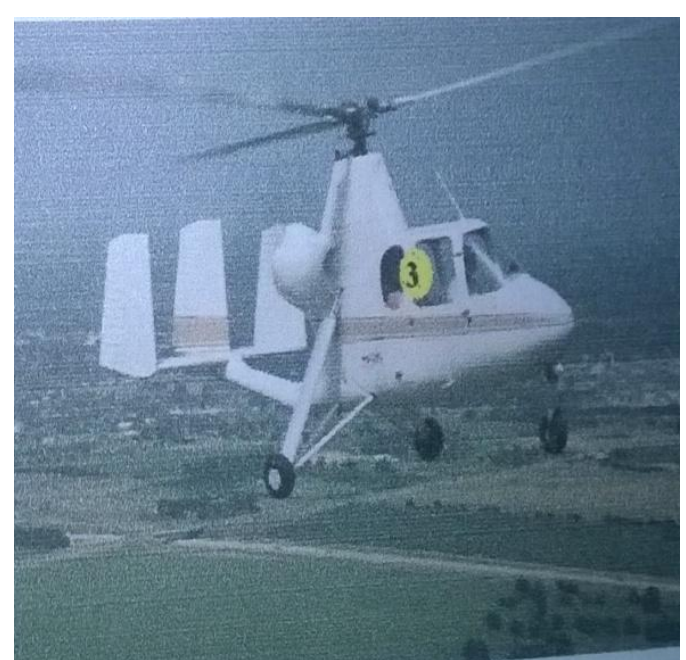

Figure 3.2.2: Cued Click Point

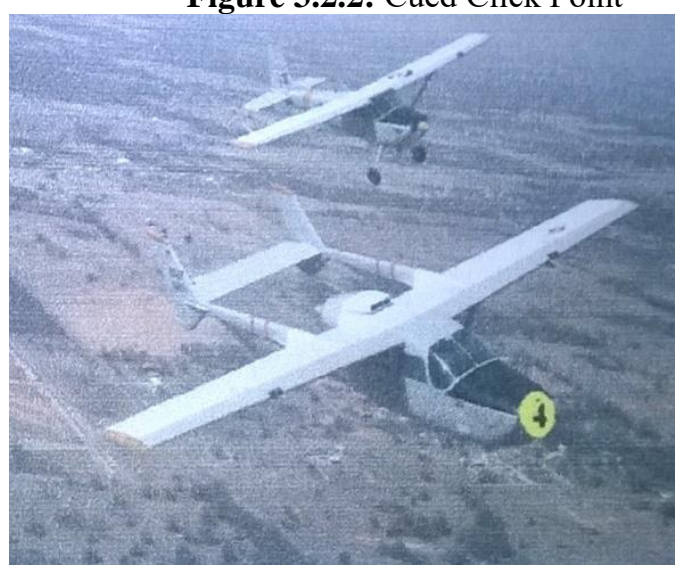

Figure 3.2.3: Cued Click Point

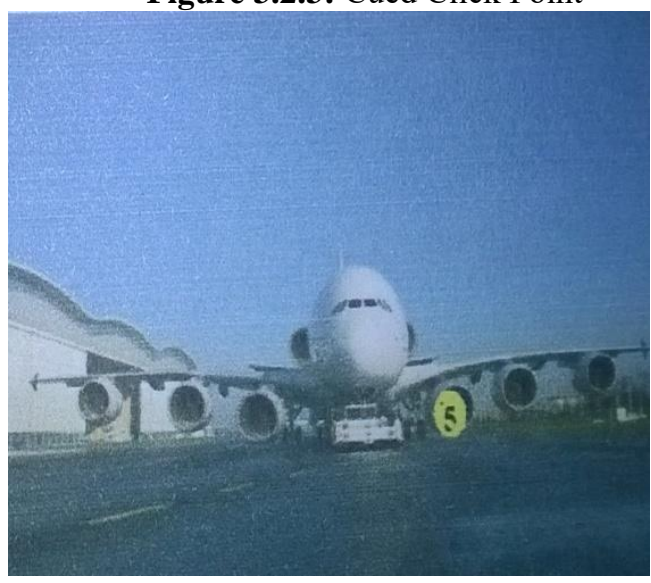

Figure 3.2.4:Cued Click Point

\subsection{Persuasive Cued Click Points(PCCP):}

In Persuasive Cued Click Points method [1],[2],[3],[4], [5] we provide small vies port area that is position on image, therefore user must select click point the view port. If user is unable to select click point within a view port then user press shuffle button.

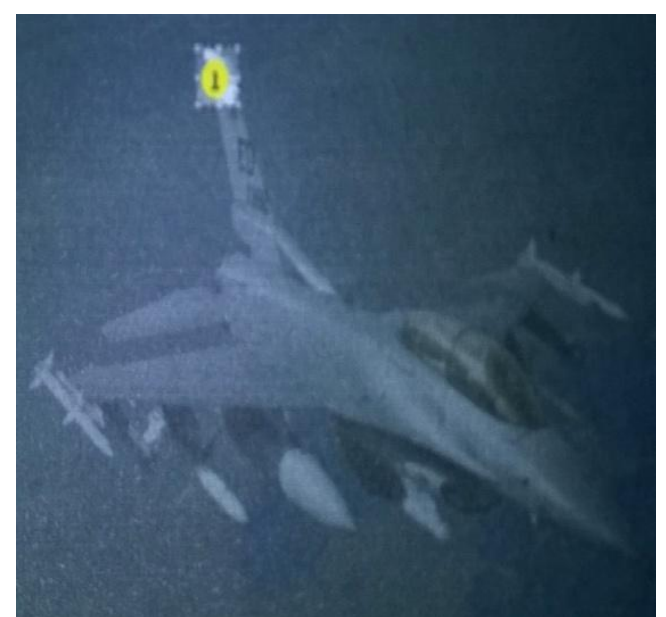

Figure 3.3.1.Persuasive Cued Click Point

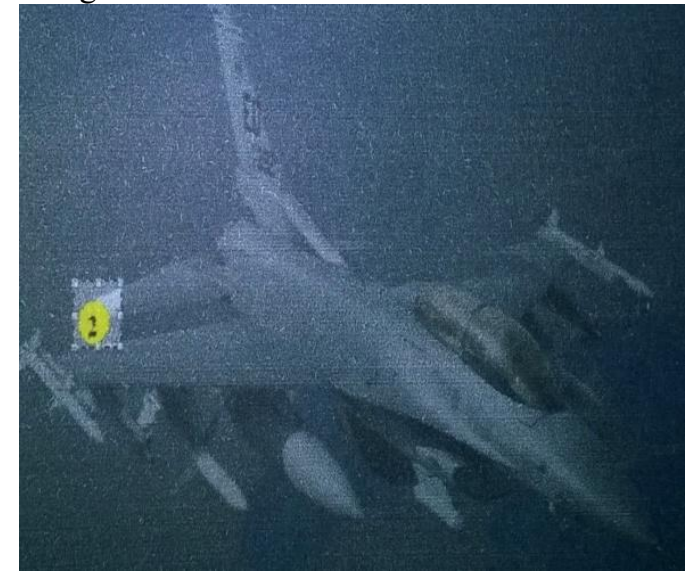

Figure 3.3.2.Persuasive Cued Click Point

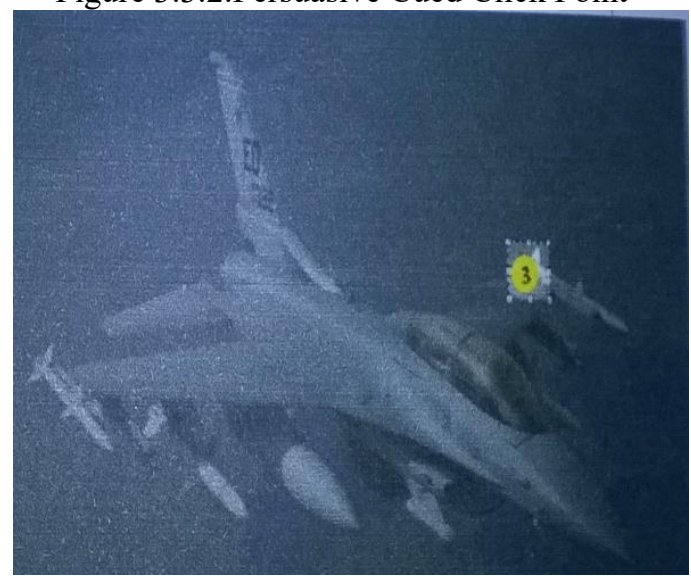

Figure 3.3.3.Persuasive Cued Click Point

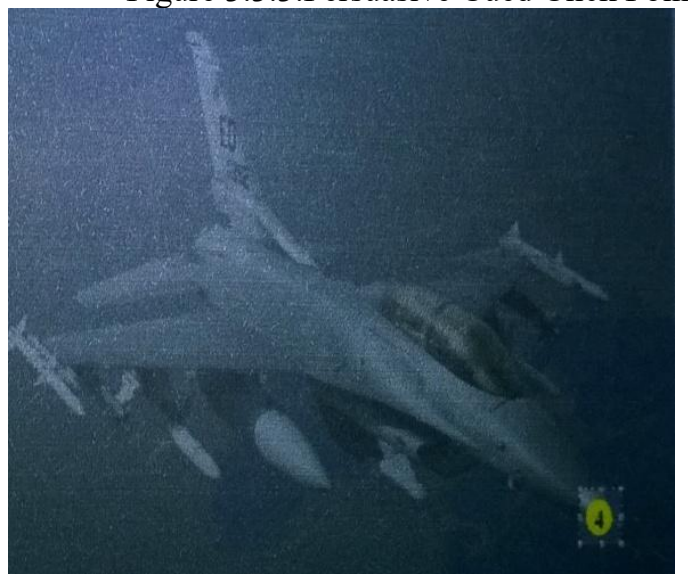

Figure 3.3.4.Persuasive Cued Click Point

Volume 4 Issue 11, November 2015 www.ijsr.net 


\section{International Journal of Science and Research (IJSR) \\ ISSN (Online): 2319-7064}

Index Copernicus Value (2013): 6.14 | Impact Factor (2014): 5.611

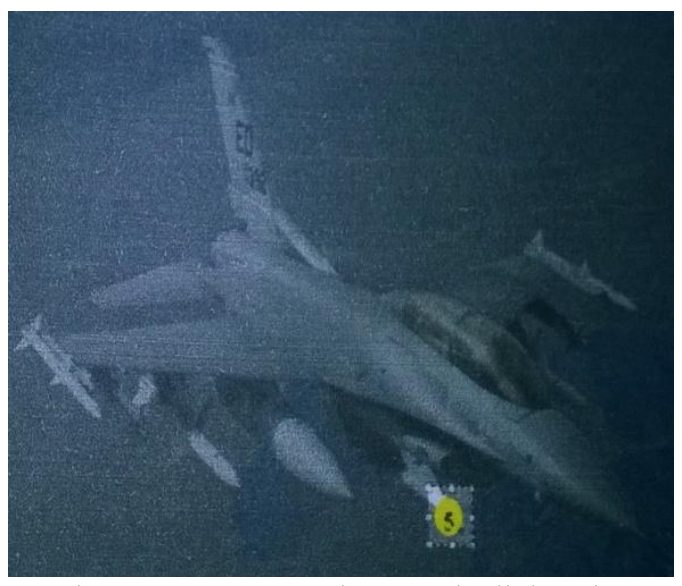

Figure 3.3.5.Persuasive Cued Click Point

\subsection{Sound}

For the registered user identification purpose in this paper we include one right click point play correct sound which is selected at the time of registration.

\section{Data Analysis}

\subsection{Graphical Analysis}

Graphical analysis simply means displaying the data in a variety of visual formats that make it easy to see patterns and identify differences among the result set. To recognize the click points $\mathrm{X}$-axis-axis are used. At the time of registration user select the click point on image is recognize by ImageClickeventArgs class. This class provide data for any event that occur when a user clicks on image based asp.net server control, such as HtmlInputImage or ImageButton server controls. While clicking an ImageButton server control causes a click event occur. At the time of login when user click on image to recognize click Math class is used. Math Class provides constant and static methods for Trigonometric, Logarithmic and other common mathematical function. Math.Abs()method returns the absolute value of a specified number.

\section{Conclusion}

This paper is usable and memorable authentication mechanism. By taking advantage of user"s ability to recognize image. Cued Click Point method has advantages over Pass Point method in terms of usability. Only one click point per image appears easier to remember than having ordered series of clicks on one image.

Persuasive cued click point ${ }^{\circ}$ s method offers more secure alternative to Pass Points and Cued Click Points methods. Persuasive Cued Click Points Method increases the work load for attackers by forcing them to conduct viewport analysis on image.

\section{Future Work}

This is a new prototype for graphical password authentication. We can enhance the prototype to transform it into a real system with good usability features.

\section{References}

1. [IJESAT] DEFENSES AGAINST LARGE SCALE

ONLINE PASSWORD GUESSING ATTACKS BY USING PERSUASIVE CLICK POINTS Abdul Rasheed. Sk ${ }^{1}$, Madhava Naidu.V ${ }^{2}$, D.sunitha ${ }^{3}$

2. PERSUASIVE CLICK POINTS BASED LARGE SCALE ONLINE PASSWORD GUESSING ATTACKS K. HARI KRISHNA

3. INTERNATIONAL JOURNAL OF COMPUTER ENGINEERING\& TECHNOLOGY (IJCET) "DEFENSES AGAINST LARGE SCALE ONLINE PASSWORD GUESSINGATTACKS BY USING PERSUASIVE CLICK POINTS" Mrs. M. A. Patel ${ }^{1}$, Ms. Y.U.Kadam ${ }^{2}$, Ms. R. Y.Thombare ${ }^{3}$, Ms. H. P. Patil $^{4}$

4. International Journal of Computer Science and Mobile Computing "Defenses against Large Scale Online Password Guessing by Using Persuasive Cued Click Points"

5. IOSR Journal of Computer Engineering (IOSR-JCE) "Online Password Guessing Attacks by Using Persuasive Click Point with Dynamic User Block" P. Kalaivizhil ${ }^{1}$, Dr. S. Thiru Nirai Senthil ${ }^{2}$ 